Routine versus clinically indicated replacement of peripheral intravenous catheters: a randomised controlled equivalence trial

Claire M Rickard, Joan Webster, Marianne C Wallis, Nicole Marsh, Matthew R McGrail, Venessa French, Lynelle Foster, Peter Gallagher, John R Gowardman, Li Zhang, Alice McClymont, Michael Whitby

Research Centre for Clinical and Community Practice Innovation, Griffith Health Institute, Griffith University, Nathan, QLD, Australia (Prof C M Rickard PhD, Prof J Webster BA, Prof M C Wallis PhD, N Marsh BN, J R Gowardman FCICM/FRACP, L Zhang PhD, Prof M Whitby PhD); Research and Development Unit, Centre for Clinical Nursing (Prof C M Rickard, Prof J Webster, N Marsh, A McClymont RN), and Department of Intensive Care Medicine (J R Gowardman), Royal Brisbane and Women's Hospital, Brisbane, QLD, Australia; Gippsland Medical School, Monash University, Churchill, VIC, Australia (M R McGrail PhD); Princess Alexandra Hospital, Woolloongabba, QLD, Australia (Prof C M Rickard, V French RN, P Gallagher MBA, Prof M Whitby); and Gold Coast Hospital, Southport, QLD, Australia (Prof M C Wallis, L Foster MN)

Correspondence to:

Prof Claire M Rickard, Research Centre for Clinical and Community Practice Innovation, Griffith Health Institute, Griffith University, Nathan, QLD 4111, Australia c.rickard@griffith.edu.au 


\section{Routine versus clinically indicated replacement of peripheral intravenous catheters: a randomised controlled equivalence trial}

\section{Summary}

Background The millions of peripheral intravenous catheters used each year are recommended for 72-96 h replacement in adults. This routine replacement increases health-care costs and staff workload and requires patients to undergo repeated invasive procedures. The effectiveness of the practice is not well established. Our hypothesis was that clinically indicated catheter replacement is of equal benefit to routine replacement.

Methods This multicentre, randomised, non-blinded equivalence trial recruited adults ( $\geq 18$ years) with an intravenous catheter of expected use longer than 4 days from three hospitals in Queensland, Australia, between May 20, 2008, and Sept 9, 2009. Computer-generated random assignment (1:1 ratio, no blocking, stratified by hospital, concealed before allocation) was to clinically indicated replacement, or third daily routine replacement. Patients, clinical staff, and research nurses could not be masked after treatment allocation because of the nature of the intervention. The primary outcome was phlebitis during catheterisation or within $48 \mathrm{~h}$ after removal. The equivalence margin was set at $3 \%$. Primary analysis was by intention to treat. Secondary endpoints were catheter-related bloodstream and local infections, all bloodstream infections, catheter tip colonisation, infusion failure, catheter numbers used, therapy duration, mortality, and costs. This trial is registered with the Australian New Zealand Clinical Trials Registry, number ACTRN12608000445370.

Findings All 3283 patients randomised (5907 catheters) were included in our analysis (1593 clinically indicated; 1690 routine replacement). Mean dwell time for catheters in situ on day 3 was 
$99 \mathrm{~h}$ (SD 54) when replaced as clinically indicated and $70 \mathrm{~h}$ (13) when routinely replaced. Phlebitis occurred in 114 of 1593 (7\%) patients in the clinically indicated group and in 114 of 1690 (7\%) patients in the routine replacement group, an absolute risk difference of $0.41 \%$ (95\% CI -1.33 to $2 \cdot 15 \%$ ), which was within the prespecified $3 \%$ equivalence margin. No serious adverse events related to study interventions occurred.

Interpretation Peripheral intravenous catheters can be removed as clinically indicated; this policy will avoid millions of catheter insertions, associated discomfort, and substantial costs in both equipment and staff workload. Ongoing close monitoring should continue with timely treatment cessation and prompt removal for complications.

Funding Australian National Health and Medical Research Council. 


\section{Routine versus clinically indicated replacement of peripheral intravenous catheters: a randomised controlled equivalence trial}

\section{Introduction}

Up to $70 \%$ of patients in acute care hospitals need a short peripheral intravenous catheter; about 200 million are used each year in the USA alone. ${ }^{1,2}$ Intravenous catheters frequently fail before the end of treatment because of irritation of the vein (phlebitis) with symptoms including pain, swelling, redness, occlusion, and a palpable venous cord. Phlebitis necessitates catheter removal and replacement. Peripheral intravenous catheter-related bloodstream infection is a less frequent but serious complication, occuring in about $0.1 \%$ of intravenous catheters or 0.5 per 1000 catheter days. ${ }^{3}$

Intravenous catheters are often needed for a week or more, but have been recommended for regular removal and replacement with a new catheter in an attempt to decrease both phlebitis and infection. Replacement requires additional needlesticks for patients, increases work for clinical staff, and contributes to insertion of intravenous catheters being the most common invasive medical procedure and therefore a substantial contributor to health-care costs. ${ }^{2}$ Routine intravenous catheter replacement no more frequently than every $72-96$ hours is currently recommended for adults by the US Centers for Disease Control and Prevention (CDC). ${ }^{4}$ By contrast, the CDC recommends not to routinely replace intravenous catheters in children, or in high-risk catheters, such as arterial, haemodialysis, and central venous catheters, for which research has shown routine replacement does not prevent infection., ${ }^{4,5}$

Modern intravenous catheters are made of low-irritant materials and might not need routine replacement. Results of well-designed observational studies have shown that longer dwell time increases daily phlebitis risk in a linear rather than exponential manner (ie, more intravenous catheter 
days overall increases risk, but later days of cannulation are not higher risk than earlier days). ${ }^{6-8}$ Thus, routine replacement of intravenous catheters might not benefit patients overall, since the same number of complications might be spread between larger numbers of devices. In 2007-10, results of four randomised controlled trials $(n=200-755)$ supported clinically indicated removal of intravenous catheters as a safe alternative to routine replacement. ${ }^{9-12}$ Despite this evidence, concerns have remained about whether abandoning this established practice would increase bloodstream infections. ${ }^{1}$ The 2011 CDC guidelines designate clinically indicated replacement of intravenous catheters as an unresolved issue, indicating that more research is needed. ${ }^{4}$

We aimed to compare the effect of extension of intravenous catheter dwell-time beyond 3 days with replacement of catheters only for clinical reasons. We postulated that patients who had intravenous catheters replaced when clinically indicated would have equivalent rates of phlebitis, and no difference in other complications, but reduced costs and number of catheter insertions, compared to patients with catheters removed every third day.

\section{Methods}

\section{Study design and participants}

We did this multicentre, non-blinded, randomised controlled equivalence trial in three universityaffiliated, government hospitals in Queensland, Australia (Royal Brisbane and Women's Hospital, Herston; Princess Alexandra Hospital, Woolloongabba; and Gold Coast Hospital, Southport). Recruitment was from May 20, 2008, until the target sample size was achieved on Sept 9, 2009.

Research nurses screened medical and surgical units daily for participants. Patients aged at least 18 years with an intravenous catheter in place and expected treatment of longer than 4 days were eligible. Exclusion criteria were bloodstream infection, planned removal of intravenous catheter 
within $24 \mathrm{~h}$, or intravenous catheter already in situ for more than $72 \mathrm{~h}$. We permitted intravenous catheters inserted in any clinical area, including the emergency department and operating room. Intravenous catheters inserted in an emergency were not eligible (hospital policies required that these be replaced within $24 \mathrm{~h}$ ). Intravenous catheters could be inserted by any nurse or doctor or by intravenous insertion teams (with no post-insertion care) in two of the hospitals. The ethics committee at each hospital and at Griffith University approved the protocol. We obtained written, informed consent from all participants before enrolment.

\section{Randomisation and masking}

Patients were randomly assigned to one of two treatment groups (simple randomisation with 1:1 ratio, no blocking, stratified by hospital). Random allocations were computer-generated on a handheld device, at the point of each patient's study entry, and thus were concealed to patients, clinical staff, and research staff until this time. Patients and clinical staff could not be masked after allocation because of the nature of the intervention. Research nurses were similarly not masked because they had to allocate patients to the treatment group and monitor the integrity of the intervention. However, laboratory staff were masked for rating of all microbiological endpoints, and a masked, independent medical rater diagnosed catheter-related infections and all bloodstream infections. We did blinded inter-rater reliability checks on a subset of phlebitis assessments.

\section{Procedures}

Patients in the clinically indicated group had their intravenous catheters removed only for completion of therapy, phlebitis, infiltration, occlusion, accidental removal, or suspected infection. Patients in the routine replacement group had their intravenous catheters replaced every third calendar day, unless clinical reasons made this impossible (eg, intravenous catheters failed before day 3 , or patient unable to be recannulated). The day 3 resite occurred at about $72 \mathrm{~h}$ (48-96 h depending on insertion 
and removal times). Investigators and research nurses had no involvement in the decision to remove intravenous catheters, or to order cultures, and did not resite the catheters. Preinsertion skin disinfection was with $2 \%$ chlorhexidine in $70 \%$ ethanol. Insyte Autoguard $30 \mathrm{~mm}$ intravenous catheters (BD Medical, Franklin Lakes, NJ, USA) and Tegaderm 1624W transparent dressings (3M, St Paul, MN, USA) were used, with dressings replaced weekly and when soiled or loose.

Chlorhexidine-impregnated sponges were not used. We placed no restriction on products administered through the intravenous catheters. Patients generally entered the study with their first intravenous catheter, and all subsequent intravenous catheters (maximum five) for a course of treatment were included. An independent data and safety monitoring committee reviewed blinded data at two a-priori defined intervals $(n=1000$ and $n=2000)$, and recommended the trial continue. Stopping rules were a greater than 2:1 ratio in either group for phlebitis or catheter-related bloodstream infection.

The primary outcome was phlebitis during catheterisation or within $48 \mathrm{~h}$ after removal. ${ }^{8,13}$ Phlebitis was two or more of the following, present simultaneously: (1) patient-reported pain or tenderness (on questioning, then palpation by the research nurse) with a severity of two or more on a ten-point scale; (2) erythema, extending at least $1 \mathrm{~cm}$ from the insertion site; (3) swelling, extending at least $1 \mathrm{~cm}$ from the insertion site; (4) purulent discharge; or (5) palpable venous cord beyond the intravenous catheter tip. All items apart from patient-reported pain or tenderness were rated by the research nurse after direct assessment of the patient, and review of clinical data. Phlebitis measures were repeated daily, and at $48 \mathrm{~h}$ after removal (by telephone if the patient had been discharged). A structured outcome assessment form was used and inter-rater reliability testing was done. A study manager visited each site at least monthly and was available to the research nurses at any time over the telephone. She audited study data for completeness and accuracy against hospital records and supervised the research nurses for compliance with study procedures. Monthly meetings were held 
by the investigators, research nurses, and the study manager to review progress and ensure consistency between sites.

Secondary endpoints were: (1) catheter-related bloodstream infection, defined as positive blood culture from a peripheral vein; clinical signs of infection (ie, fever, chills, or hypotension); no other apparent source for the bloodstream infection except the intravenous catheter (in situ within $48 \mathrm{~h}$ of the bloodstream infection); and a colonised intravenous catheter tip culture with the same organism as identified in the blood; ${ }^{14}(2)$ all-cause bloodstream infections, defined as any positive blood culture drawn while intravenous catheter in situ or for $48 \mathrm{~h}$ after removal; (3) local venous infection, defined as organisms grown from purulent discharge or vein segment with no evidence of associated bloodstream infection; ${ }^{14}$ (4) colonisation of intravenous catheter tip, with more than 15 colonyforming units ${ }^{15}$ (substudy of a convenience sample of $5 \%$ of patients, ie, catheters that were removed on days and times that the research nurses were available to take cultures); (5) infusion failure, defined as any premature removal of intravenous catheter before end of treatment, other than routine replacement-includes phlebitis, infiltration, occlusion, accidental removal, and catheter-related bloodstream infections; (6) number of intravenous catheters needed per patient for course of treatment; (7) overall duration of intravenous therapy (all intravenous catheters) per patient (h); (8) costs per patient for the course of intravenous therapy, based on equipment required for insetion and removal of intravenous catheter ${ }^{12}$ with prices from negotiated hospital supply contract rate; and (9) mortality with intravenous catheter in situ or within $48 \mathrm{~h}$ of removal, collected from hospital records. Hospital costs were set at AU\$25.13 for intravenous catheter plus administration set, burette, and fluid bag; $\$ 21.83$ for intravenous catheter plus administration set and fluid bag; $\$ 12.73$ for intravenous catheter plus end cap; and $\$ 0.37$ gauze and tape for removal; and staff time (observed rates of $14.5 \mathrm{~min}$ per insertion and $4.5 \mathrm{~min}$ per removal at fixed industrial award wages for registered nurses of $\$ 32.93 / \mathrm{h}$ ), junior [ $\$ 45.96 / \mathrm{h}$ ] and senior [ $\$ 67.16 / \mathrm{h}$ ] medical staff). Catheters 
inserted by intravenous insertion teams were costed at registered nurse rates; intravenous catheters inserted in an operating theatre or radiology suite were costed at senior medical officer rates, and other insertions were costed at junior medical officer to registered nurse rates in a 3:1 ratio (on the basis of internal observations). We did not include the cost of treating complications associated with intravenous catheters (since treatment typically consisted only of removal and replacement of the affected intravenous catheter, which was already accounted for in the cost calculations).

\section{Statistical analyses}

We used a two-sided design to test equivalence between groups. The sample size was calculated to detect equivalence at $4 \%$ phlebitis ${ }^{11}$ (equivalence margin $3 \%$ ) with $5 \%$ significance and more than 95\% power. This determined a total sample of 3000 patients, plus 300 to allow for attrition. Data were entered directly into a purpose-built Microsoft Access (version 97) database. We used SPSS (version 18) and StataSE (version 10) for analyses.

Baseline characteristics of patients and catheters were described by group. The primary analysis was by intention to treat, including all patients in their randomised group. First, we calculated relative incidence rates of phlebitis and absolute rate differences per 100 catheters and per 100 patients, and we then used two-sided Fisher's exact test to assess equivalence in risk between groups. Second, we calculated hazard rates per 1000 catheter hours, with 95\% CI, with a Cox proportional hazards model (assumptions were checked) to summarise the effect of intervention per patient (including all intravenous catheters per patient). Finally, we used Kaplan-Meier survival curves to compare rates of time until first phlebitis per patient between groups.

We also did a per-protocol analysis. Specifically, we analysed the first intravenous catheter per patient remaining in situ on day 3 that was treated as per the random allocation. Rates per 1000 days 
for this analysis consisted of aggregated rate comparisons (incident rate ratios) instead of hazard ratios (HR, we did not compare groups for survival from phlebitis beyond day 3 , since in this analysis all intravenous catheters in the routine resite group were removed on day 3). We calculated intravenous catheter dwell times from this analysis since only first catheters were assessed prospectively as required for 4 days or more, and to estimate the difference in catheterisation that clinically indicated removal would achieve. We retrospectively assessed power to detect equivalence with per-protocol analysis.

We compared patient-level costs and the number of peripheral intravenous catheters used per patient with arithmetic means and the independent sample $t$ test; we used bootstrapping with 1000

replications to calculate $95 \% \mathrm{CI}$ for costs. ${ }^{16} \mathrm{We}$ collected resource-use data as how many catheters a patient had fitted and removed, and the costs associated with the insertion setting (eg, by staff in the operating theatre $v s$ the medical and surgical wards). We compared overall treatment time (all intravenous catheters) between groups with the median, quartile, range, and Mann-Whitney test. We deemed $\mathrm{p}$ values less than $0 \cdot 05$ to be significant.

This trial is registered with the Australian New Zealand Clinical Trials Registry, number ACTRN12608000445370.

\section{Role of the funding source}

The sponsor had no involvement in the design and conduct of the study; collection, management, analysis, and interpretation of the data; and preparation, review, or approval of the report. The corresponding author had full access to study data and final responsibility in submitting the report for publication. 


\section{Results}

Of 3379 eligible patients, 3283 were enrolled, and no patient withdrew consent (figure 1). In total, we studied 5907 intravenous catheters and 17412 catheter days (clinically indicated 8693 days; routine replacement 8719 days). Tables 1 and 2 show patient and catheter characteristics at baseline. Protocol adherence was $85 \%$ in the clinically indicated group and $70 \%$ in the routine replacement group (figure 1). Of 1593 patients in the clinically indicated group, 1351 had 1844 intravenous catheters in place on day 3 , and removed for clinical reasons; of 1690 patients randomised to routine replacement, 1186 had 1744 intravenous catheters removed on day 3. Mean intravenous catheter dwell time was $99 \mathrm{~h}$ (range 48-561 h) in the clinically indicated group and $70 \mathrm{~h}$ (range 48-96 h) in the routine replacement group (table 1).

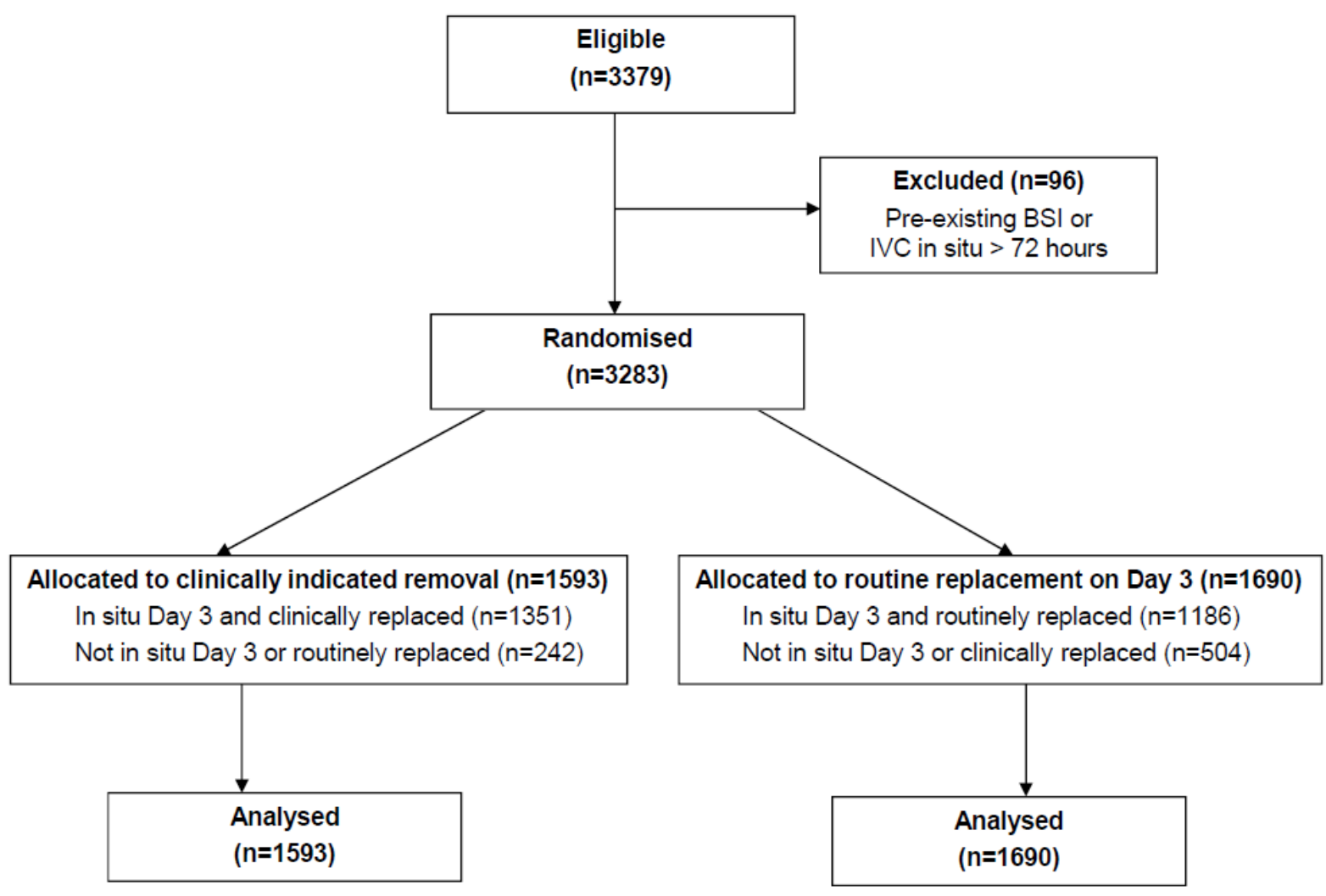

Figure 1. Study profile of patient flow 
Table 1. Baseline demographics and clinical characteristics of patients

\begin{tabular}{lcc}
\hline & $\begin{array}{c}\text { Clinically indicated } \\
(\mathbf{n = 1 5 9 3})\end{array}$ & $\begin{array}{c}\text { Routine replacement } \\
(\mathbf{n}=\mathbf{1 6 9 0})\end{array}$ \\
\cline { 2 - 3 } Intravenous catheter dwell time (h)* & $70(13)$ \\
Mean (SD) & $99(54)$ & $70(57-77)$ \\
$\quad$ Median (IQR) & $84(64-118)$ & $55 \cdot 0(18 \cdot 4)$ \\
Age (years) & $55 \cdot 1(18 \cdot 6)$ & $1034(61 \%)$ \\
Men & $1022(64 \%)$ & \\
Type of admission & & $331(20 \%)$ \\
$\quad$ Medical & $292(18 \%)$ & $1359(80 \%)$ \\
$\quad$ Surgical & $1301(81 \%)$ & \\
Comorbidities & & $411(24 \%)$ \\
$\quad$ None & $387(24 \%)$ & $372(22 \%)$ \\
One & $350(22 \%)$ & $907(54 \%)$ \\
$\quad$ Two or more & $856(54 \%)$ & $244(14 \%)$ \\
Present wound & $256(16 \%)$ & $116(7 \%)$ \\
infection & & $37(2 \%)$ \\
Wound drain & $95(6 \%)$ & \\
Stoma & $27(2 \%)$ &
\end{tabular}

Data are mean (SD) or $\mathrm{n}(\%)$. *Per protocol, $\mathrm{N}=1351$ clinically indicated; $\mathrm{N}=1186$ routine replacement.

Table 2. Characteristics of peripheral intravenous catheters (per intravenous catheter analysis)

$\begin{array}{cc}\text { Clinically indicated }_{(\mathbf{n}=2692)}^{*} & \text { Routine replacement }^{\dagger} \\ (\mathbf{n}=3215)\end{array}$

Inserted by

$\begin{array}{lll}\text { General clinical staff } & 1656(62 \%) & 1856(58 \%) \\ \text { IV insertion service } & 1002(38 \%) & 1320(42 \%)\end{array}$

Catheter Gauge

$\begin{array}{lcc}\leq 18 & 430(16 \%) & 481(15 \%) \\ 20 & 1517(57 \%) & 1746(54 \%) \\ \geq 22 & 736(27 \%) & 984(31 \%)\end{array}$

Insertion in

Ward

$2002(74 \%)$

$2467(77 \%)$ 
Emergency

Operating theatre or radiology

Other

Skin integrity

Poor

Fair

Good

Vein quality

Poor

Fair

Good

Insertion side

Left side

Right side

Insertion site

Cubital fossa

Hand

Inner forearm

Lower forearm

Mid forearm

Outer forearm

Wrist

Upper arm

Other

Prescribed treatment

Oral antibiotic

Intravenous antibiotic

Intravenous crystalloid

Intravenous potassium

Intravenous antipyretic

Intravenous cortisone

Other intravenous
$276(10 \%)$

$305(10 \%)$

$361(13 \%)$

$53(2 \%)$

$57(2 \%)$

$116(4 \%)$

$153(5 \%)$

767 (29\%)

$849(26 \%)$

1809 (67\%)

$2213(69 \%)$

$499(16 \%)$

$1154(36 \%)$

$928(35 \%)$

1562 (49\%)

$1383(51 \%)$

$1390(52 \%)$

$1616(50 \%)$

$1301(48 \%)$

$1599(50 \%)$

$394(12 \%)$

$726(23 \%)$

$346(11 \%)$

$662(21 \%)$

$532(17 \%)$

$182(7 \%)$

$164(5 \%)$

$69(3 \%)$

$81(3 \%)$

$201(8 \%)$

$248(8 \%)$

$36(1 \%)$

$62(2 \%)$

$$
84(3 \%)
$$

$88(3 \%)$

1835 (68\%)

2235 (70\%)

2668 (99\%)

3180 (99\%)

$222(8 \%)$

247 (8\%)

$160(6 \%)$

$158(5 \%)$

$92(3 \%)$

$73(2 \%)$

$1158(43 \%)$

$1327(41 \%)$ drugs

In some instances, total numbers are not 2692 or 3215 per group because of missing data. Some totals do not add to $100 \%$ because of rounding. *In 1593 patients. $\dagger$ In 1690 patients. 
Phlebitis inter-rater reliability testing across the three hospitals (248 blinded paired observations) showed $98 \%$ agreement, with a Cohen's $\kappa$ of 0.33 . In the primary analysis, in both groups $7 \%$ of patients had phlebitis (table 3), with an absolute risk difference (ARD) of $0.41 \%$ (95\% CI -1.33 to $2 \cdot 15)$, which was within the predefined equivalence margin of $3 \%$. Therefore we accepted the equivalence hypothesis. All comparisons of phlebitis occurrence between study groups were equivalent including per patient ( $\mathrm{p}=0 \cdot 64$, table 3 ) and per 1000 catheter days ( $\mathrm{p}=0 \cdot 67$, table 3 ), and on survival analysis ( $\mathrm{p}=0.96$, figure 2$)$. The per-protocol analysis $(\mathrm{n}=2537)$ had consistent results with the primary analysis with ARD $0 \cdot 70 \%$ (95\% CI $-0 \cdot 88$ to $2 \cdot 28$, table 3 ); this comparison had $90 \%$ power $(\mathrm{p}=0 \cdot 05)$ to detect equivalence (margin $3 \%)$ at the recorded occurrence of phlebitis of $5.5 \%$. 


\section{Clinically \\ Routine \\ Risk (95\% CI) p value \\ indicated replacement}

$(n=1593)$ $(\mathbf{n}=1690)$

\section{Primary outcome, intention-to-treat analysis}

Phlebitis per patient, $\mathrm{n} \quad 114(7 \%)$

$(\%)$

Phitis/1000

$$
13 \cdot 08
$$

$(10 \cdot 68-15 \cdot 48)$

days* $(95 \% \mathrm{CI})$
$114(7 \%)$

$13 \cdot 11$

$(10 \cdot 71-15 \cdot 52)$
RR $1.06(0 \cdot 83$

$0 \cdot 64$ to $1 \cdot 36)$; ARD $0.41 \%$ $(-1 \cdot 33$ to $2 \cdot 15)$ HR $0.94(0.73$ $0 \cdot 67$ to $1 \cdot 23)$

\section{Primary outcome, per-protocol analysis*}

Phlebitis per patient

$63 / 1351(4 \cdot 66 \%)$

$47 / 1186(4 \%)$
RR 1.18

$0 \cdot 39$ (0.81 to $1 \cdot 70)$;

ARD $0.70 \%$

(-0.88 to $2 \cdot 28)$

Phlebitis/1000

$$
11 \cdot 4
$$

intravenous catheter days

(95\% CI)

$(8 \cdot 6-14 \cdot 2)$

$13 \cdot 8$

$(9 \cdot 9-17 \cdot 8)$

$(0 \cdot 56$ to $1 \cdot 23)$

HR $0.99(0.89 \quad 0.87$

to $1 \cdot 11)$

HR $1.06(0.89 \quad 0.51$

to $1 \cdot 27)$

Occlusion

$344(39 \cdot 5)$

$344(39 \cdot 6)$

Accidental removal

$166(19 \cdot 0)$

$159(18 \cdot 3)$

CRBSI

$$
0(0)
$$

All BSI

$$
4(0 \cdot 46)
$$

Venous (local) infection $\$$

$$
0
$$

\section{0}

$$
\text { HR 0.92 (0.79 }
$$

$$
\text { to } 1.07)
$$

HR $0.98(0.79 \quad 0.88$

$$
\text { to } 1.23 \text { ) }
$$

HR $0.46(0 \cdot 14$

$0 \cdot 19$

$$
\text { to } 1 \cdot 48)
$$

$$
\text { Mortality, n (\%)§ }
$$

$$
4(<1 \%)
$$

$0 \cdot 93$

$\mathrm{ARD}=$ absolute risk difference. $\mathrm{BSI}=$ bloodstream infection. $\mathrm{CRBSI}=$ catheter-related bloodstream infection. $\mathrm{HR}=$ hazard ratio. $\mathrm{IRR}=$ incident rate ratio. $\mathrm{RR}=$ relative risk. *First catheter per patient only. $\dagger$ Combined endpoint of phlebitis, infiltration, occlusion, accidental removal, and CRBSI. $\$$ Risk and $\mathrm{p}$ value inestimable because of 0 incidence. $\S I n$ all cases, mortality was unrelated to intravenous catheter treatment. 


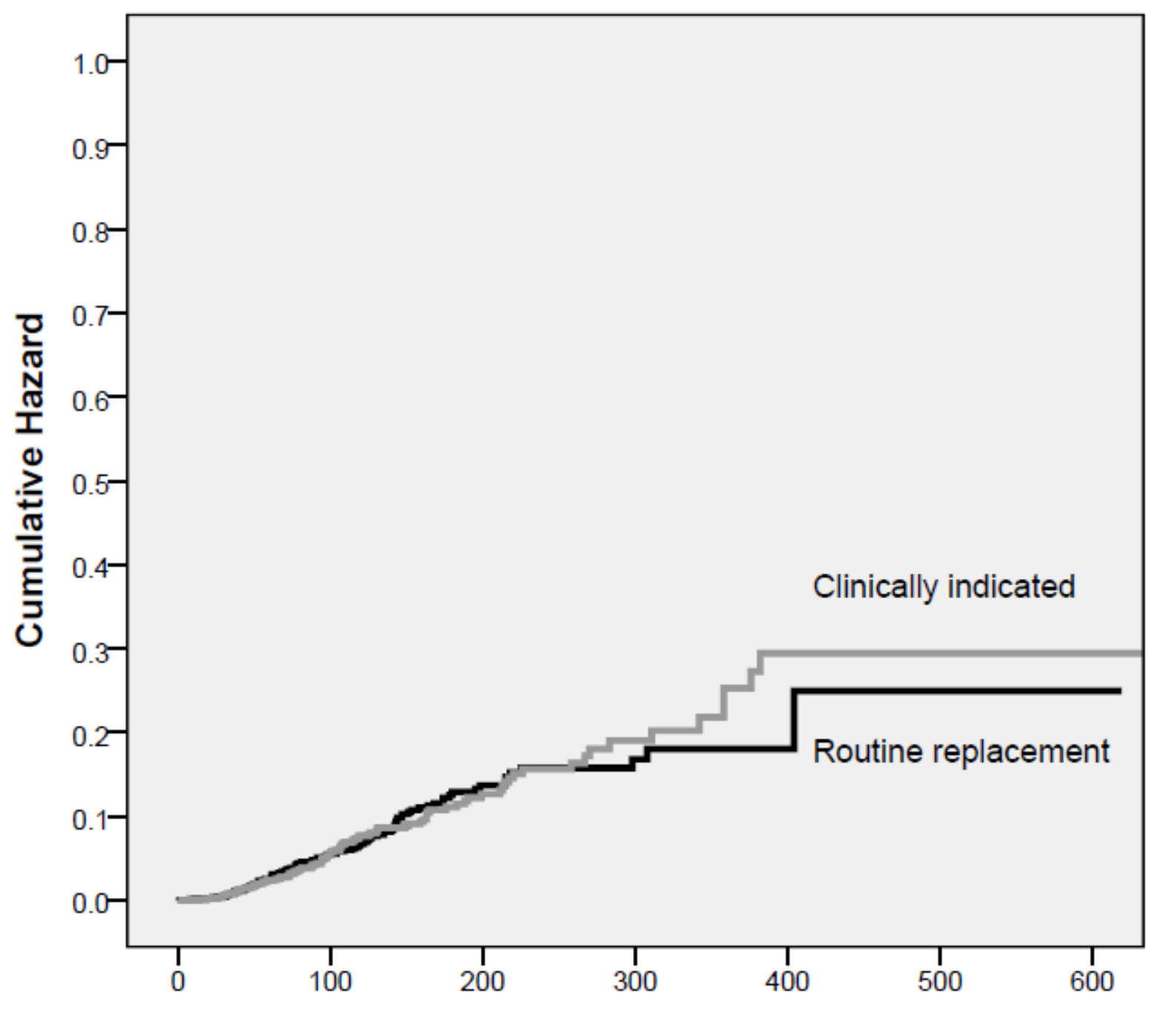

Cumulative time (hours) up to first phlebitis episode

$\begin{array}{llllll}\text { Clin. indicated } & 66 / 1590 & 31 / 739 & 10 / 230 & 6 / 91 & 1 / 44 \\ \text { Routine replace } & 70 / 1686 & 37 / 743 & 5 / 231 & 1 / 90 & 1 / 15\end{array}$

Figure 2. Kaplan-Meier analysis of survival from phlebitis per patient Includes all catheters per patient, log-rank $\mathrm{p}=0 \cdot 96$.

No patient had a venous (local) infection and groups were equivalent for all-cause bloodstream infections, and catheter colonisation. Only one patient had a catheter-related bloodstream infection and this patient was in the routine replacement group. Overall, we identified 15 positive blood cultures from 13 patients (four patients in the clinically indicated group; nine patients in the routine replacement group [two patients had two separate bloodstream infections]). All bloodstream 
infections in the clinically indicated group were Gram positive organisms, whereas Gram positive and negative organisms were similarly represented within the routine replacement group (table 4). In the substudy ( $\mathrm{n}=298)$, intravenous catheter colonisation rates did not differ between groups (clinically indicated $13 \cdot 0 / 1000$ catheter days [eight of $143,6 \%$ of intravenous catheters]; routine replacement $12 \cdot 4 / 1000$ catheter days [six of $155,4 \%$ of intravenous catheters]; HR $1 \cdot 05$ [95\% CI $0 \cdot 32-3 \cdot 68])$. None of these colonised tips were associated with a bloodstream infection.

Rates of infiltration, occlusion, accidental removal, total infusion failure, and in-hospital mortality were all equivalent between groups (table 3). The groups had equivalent overall duration of intravenous treatment; however, the clinically indicated group required significantly fewer intravenous catheters per patient, with significantly reduced hospital costs (both $p<0 \cdot 0001$, table 5). No serious adverse events were related to the trial intervention. 
Table 4. Microbiological and clinical information for 15 positive blood cultures from 13 patients

\begin{tabular}{|c|c|c|c|c|c|}
\hline & Study Group & $\begin{array}{l}\text { Clinical signs of } \\
\text { sepsis }\end{array}$ & $\begin{array}{l}\text { Antimicrobial } \\
\text { treatment } \\
\text { started }\end{array}$ & $\begin{array}{c}\text { Matched positive } \\
\text { intravenous } \\
\text { catheter tip culture }\end{array}$ & Other matched positive culture \\
\hline Staphylococcus aureus & $\begin{array}{l}\text { Clinically } \\
\text { indicated }\end{array}$ & Yes & Yes & No & No \\
\hline$S$ aureus & $\begin{array}{l}\text { Clinically } \\
\text { indicated }\end{array}$ & Yes & Yes & No & No \\
\hline $\begin{array}{l}\text { Coagulase negative } \\
\text { staphylococci }\end{array}$ & $\begin{array}{l}\text { Clinically } \\
\text { indicated }\end{array}$ & No & No & No & No \\
\hline$S$ epidermidis & $\begin{array}{l}\text { Clinically } \\
\text { indicated }\end{array}$ & No & No & No & No \\
\hline Pseudomoas aeruginosa & $\begin{array}{l}\text { Routine } \\
\text { replacement }\end{array}$ & Yes & Yes & No & Urine: Pseudomonas aeruginosa \\
\hline $\begin{array}{l}\text { Coagulase negative } \\
\text { staphylococci }\end{array}$ & $\begin{array}{l}\text { Routine } \\
\text { replacement }\end{array}$ & Yes & Yes & No & $\begin{array}{c}\text { Wound: Coagulase negative } \\
\text { staphylococci }\end{array}$ \\
\hline$S$ aureus & $\begin{array}{l}\text { Routine } \\
\text { replacement }\end{array}$ & Yes & Yes & No & Wound: $S$ aureus \\
\hline Escherichia coli & $\begin{array}{l}\text { Routine } \\
\text { replacement }\end{array}$ & Yes & Yes & No & No \\
\hline Enterobacter cloacae $^{*}$ & $\begin{array}{l}\text { Routine } \\
\text { replacement }\end{array}$ & Yes & Yes & $\begin{array}{c}\text { Enterobacter cloacae } \\
>100 C F U\end{array}$ & No \\
\hline E coli $\dagger$ & $\begin{array}{l}\text { Routine } \\
\text { replacement }\end{array}$ & Yes & Yes & No & Urine: Escherichia coli \\
\hline $\begin{array}{l}\text { Coagulase negative } \\
\text { staphylococci } \dagger\end{array}$ & $\begin{array}{l}\text { Routine } \\
\text { replacement }\end{array}$ & No & No & No & No \\
\hline Bacteroides fragilis $\ddagger$ & $\begin{array}{l}\text { Routine } \\
\text { replacement }\end{array}$ & Yes & Yes & No & No \\
\hline $\begin{array}{l}\text { Coagulase negative } \\
\text { staphylococcił }\end{array}$ & $\begin{array}{l}\text { Routine } \\
\text { replacement }\end{array}$ & No & No & No & No \\
\hline $\begin{array}{l}\text { Coagulase negative } \\
\text { staphylococci }\end{array}$ & $\begin{array}{l}\text { Routine } \\
\text { replacement }\end{array}$ & Yes & Yes & No & No \\
\hline Klebsiella oxytoca & $\begin{array}{l}\text { Routine } \\
\text { replacement }\end{array}$ & Yes & Yes & No & No§ \\
\hline
\end{tabular}


Table 5. Comparison of resource use and costs by treatment group (per patient analysis)

\begin{tabular}{|c|c|c|c|c|}
\hline & $\begin{array}{l}\text { Clinically } \\
\text { indicated } \\
(\mathrm{n}=1593)\end{array}$ & $\begin{array}{l}\text { Routine } \\
\text { replacement } \\
(n=1690)\end{array}$ & $\begin{array}{l}\text { Difference } \\
(95 \% \mathrm{CI})\end{array}$ & p value \\
\hline Duration of therapy $(\mathrm{h})^{*}$ & $\begin{array}{c}98 \\
(69-161)\end{array}$ & $\begin{array}{c}96 \\
(66-162)\end{array}$ & - & $0 \cdot 12$ \\
\hline $\begin{array}{l}\text { Intravenous catheters } \\
\text { used }\end{array}$ & $1 \cdot 7(1 \cdot 0)$ & $1 \cdot 9(1 \cdot 2)$ & $\begin{array}{c}0 \cdot 21 \\
(0 \cdot 13-0 \cdot 29) \\
\end{array}$ & $<0 \cdot 0001$ \\
\hline Costs of therapy (AU\$) $\dagger$ & $\begin{array}{l}\text { AUD } \$ 61 \cdot 66 \\
(\$ 39 \cdot 46)\end{array}$ & $\begin{array}{l}\text { AUD } \$ 69 \cdot 24 \\
(\$ 43 \cdot 45)\end{array}$ & $\begin{array}{c}\$ 7 \cdot 58 \\
(\$ 4 \cdot 78-\$ 10 \cdot 38)\end{array}$ & $<0 \cdot 0001$ \\
\hline
\end{tabular}

Data are median (IQR) or mean (SD). *Cumulative of all intravenous catheters per patient. $\dagger 2011$ cost.

\section{Discussion}

Phlebitis occurred in $7 \%$ of patients when intravenous catheters were removed when clinically indicated and when they were removed routinely every 3 days. The absolute difference was small $(0.41 \%)$ and within the prestated $3 \%$ equivalence margin. We accepted the equivalence hypothesis and results were consistent across all analyses including per patient, per protocol, per catheter, and per 1000 catheter days. Likewise, study groups had equivalent occurrence of catheter-related bloodstream infections and all-cause bloodstream infections, with no local infections in either group.

Catheter-related bloodstream infections were rare in our study at one per $3283(0.03 \%)$ patients or one per $5907(0.02 \%)$ catheters. This finding is reassuring, with no suggestion that clinically indicated replacement increased risk of bloodstream infection; this is a major piece of evidence that routine removal is not warranted. Our results confirm the low occurrence of catheter-related bloodstream infections in peripheral intravenous catheters identifiied in previous prospective studies of none in $2088,{ }^{17}$ none in $1054,{ }^{13}$ and none in 6538 intravenous catheters ${ }^{18}$.

Consideration of the pathogenesis of catheter-related bloodstream infection might assist in understanding of our results. Such infections are initially related to the insertion procedure (poor hand hygiene or skin preparation) with later infections caused by colonisation along the skin tract, or 
contaminated hubs or fluids. ${ }^{19}$ Although routine replacement of intravenous catheters theoretically could reduce later infections, conversely it exposes the patient to the contamination risk of another insertion procedure. One study reported $16 \%$ of central catheter tips as already colonised immediately after insertion, ${ }^{20}$ and initial contamination might also be common for intravenous catheters. Our substudy rate of 5\% tip colonisation with no associated bloodstream infections is consistent with results of previous studies showing colonisation as a poor predictor of infection. ${ }^{6,13}$ With the role of surface preconditioning and biofilm development now attaining increased prominence, new molecular-based diagnostics might offer improved insights. ${ }^{21}$

We recruited 3283 patients with intravenous catheters who were predicted by the research nurses at enrolment to require intravenous treatment for more than 4 days. However, this was a pragmatic clinical trial: we did not expect that all patients would actually have intravenous catheters remaining in situ for this length of time, and we recruited additional patients to allow for this attrition. Ultimately, only 2537 patients (77\%) adhered to the protocol, and adherence was disproportionately lower in the routine replacement group than in the clinically indicated group (70\% vs $85 \%)$ because for staff to always replace catheters on day 3 was more difficult than leaving them in situ in the clinically indicated group. The per-protocol analysis showed that even in patients with intravenous catheters in situ on day 3, and when clinically indicated or routine replacement was consistently applied, phlebitis was equivalent between groups, with adequate statistical power for this analysis.

Intravenous catheters are already frequently left in place beyond the currently recommended 72-96 $\mathrm{h}$, typically as the result of a complex clinical judgement, rather than a policy violation. ${ }^{22,23}$ Prospective studies ${ }^{23-25}$ report $21-62 \%$ of intravenous catheters remain in situ beyond the routine time for removal, and they are usually left for appropriate reasons-eg, treatment soon to be completed, poor veins, or no available staff to cannulate. ${ }^{22-25}$ The CDC itself tempers its $72-96 \mathrm{~h}$ 
replacement recommendations with "if sites for venous access are limited and no evidence of phlebitis or infection is present, intravenous catheters can be left in place for longer". ${ }^{14}$ Thus, a change to policies of clinically indicated removal of intravenous catheters might not be very far from the current real-world approach that occurs despite policies and recommendations.

Our data predict how intravenous catheter dwell times and numbers of intravenous catheters used would change with a clinically indicated replacement approach. Intravenous catheter use will be extended, on average, by a little more than 1 day, with the IQR showing that about a quarter will remain in use for longer than 5 days. The longest intravenous catheter duration we recorded was 3 weeks, without complications. Importantly, our study was not testing intravenous catheter dwell for short versus lengthy periods per se (because we did not force intravenous catheters to remain in situ for set periods), but rather whether the policy of routine replacement reduces complications. Our data strongly suggest that routine replacement does not, but rather causes many unnecessary invasive procedures. The average reduction of 0.2 intravenous catheters per patient inserted in the clinically indicated group meant a number needed to treat of 5; that is, under a clinically indicated removal policy, one in every five patients will avoid an unecessary procedure.

Because globally a high number of patients need intravenous catheters, clinically indicated replacement would have worldwide effects on health-care costs each year. Of the 200 million catheters estimated to be inserted each year in the USA alone ${ }^{1}$, if even $15 \%$ are needed for more than 3 days, then a change to clinically required replacement would prevent up to 6 million unecessary intravenous catheter insertions, and would save about 2 million hours of staff time, and up to US\$60 million in health costs each year for that country alone. These savings could then be redirected to other health interventions with better evidence of effectiveness than routine replacement. 
Routine replacement of intravenous catheters has been practiced for four decades, during which time catheter materials have changed from steel, polyvinylchloride, polyethylene, and tetrafluoroethylenehexafluoropropylene to more biocompatible polyurethane. ${ }^{13,26}$ This change might partially explain why intravenous catheters can now be tolerated for longer periods than previously and why our $7 \%$ phlebitis occurrence was lower than that reported with other catheter materials, ${ }^{6-8,13}$ but consistent with rates of $1-7 \%$ reported with polyurethane intravenous catheters. ${ }^{11,18,27-31}$ Our rates might have been somewhat lowered by the $40 \%$ of intravenous catheters placed by intravenous insertion teams, although our teams did no post-insertion care, the main factor by which such teams are believed to reduce complications. ${ }^{32}$ Our phlebitis occurrence was also affected by a strict definition whereby pain or tenderness was one criterion rather than two, and many criteria were quantitatively restricted, compared to other definitions. Similarly, our identification of catheter-related bloodstream infections was affected by the stringent CDC definition; we required confirmed microbiological evidence of the intravenous catheter as the source of infection. Our findings might not be generalisable to settings where the incidence of phlebitis or catheter-related bloodstream infection is high. 


\section{Panel: Research in context}

\section{Systematic review}

We searched the Medline and CINAHL databases for studies comparing any duration of peripheral intravenous catheter replacement with any other duration of peripheral catheter replacement. Our search terms were "peripheral", "intravenous", "catheter/device/cannula", "replacement", "removal", "resite", "timing", “duration", "routine", "phlebitis", and "infection". The search was not restricted by date or language. We also searched reference lists of articles identified by this strategy, and updated the search during write-up of our study. The date of the last search was May 14, 2012. Previous studies were smaller than ours and did not provide a conclusive answer as to whether clinically indicated replacement is equivalent to routine replacement. Some were limited to catheters inserted in particular groups of patients, and outcome measures were often composites. Our recent systematic review $^{35}$ summarised six trials (including unpublished interim data from this trial) with a total of 3455 patients and showed no difference in phlebitis or bloodstream infection between routine and clinically indicated replacement groups. We concluded that a large trial focusing on phlebitis as the primary outcome was needed.

\section{Interpretation}

Our study was powered to answer the question of equivalence in phlebitis for clinically indicated replacement versus routine third daily replacement of peripheral intravenous catheters in general hospital patients. Bloodstream infections were confirmed as a rare complication in patients with peripheral catheters and did not differ between the groups. Our results confirm previous smaller studies' findings that clinically indicated peripheral intravenous catheter replacement is safe.

The major strengths of this study were the processes used to eliminate selection, allocation, and detection bias, and the large sample size with $100 \%$ follow-up for the primary endpoint. We included a broad range of hospital patients and did not restrict intravenous catheters by their use or by inserter. 
Most patients had several comorbidities and received vein-irritant drugs such as intravenous antibiotics. Most catheters in our sample were inserted by medical and nursing staff, not intravenous teams, and we included those inserted in general wards, emergency, or operating departments. These factors promote generalisability to other acute, complex hospital populations. Our results do not apply to intravenous catheters inserted under emergency conditions, where aseptic insertion is not achieved because such insertions were not included in our study.

The study's main limitation was its non-blinded design. Blinding was not possible because of the obvious nature of catheter placement to patients and staff. We considered using a second set of blinded research nurses for phlebitis assessments, but this procedure would be prohibitively expensive, in view of the large sample and daily measures. We recorded high phlebitis inter-rater agreement rates in a blinded substudy, suggesting our approach was acceptable. The phlebitis measures involved some subjectivity but that was reduced by use of a structured phlebitis instrument, continued training, and audit of research nurses. Patient-reported ratings were only one item (pain or tenderness) on the five-item list, and because two items were required concurrently for diagnosis, patients' perceptions alone were unable to affect phlebitis rates. Another limitation was our inability to culture all intravenous catheter tips because of restrictions of the hospital laboratories and budget. Instead we monitored all tips cultured on clinical indication, a method that produces much the same identification of catheter-related bloodstream infections, ${ }^{3,19}$ and undertook a substudy. Additionally, we could not use catheter-related bloodstream infection as the primary endpoint since rates with intravenous catheters are typically close to $0 \%$.

Worries about phlebitis and bloodstream infections have sustained routine replacement policies, with these two complications generally the focus of intravenous catheter literature, yet our data show that the more frequent reasons for modern catheter failure are infiltration, occlusion, and accidental 
removal. In total, nearly $30 \%$ of intravenous catheters had some form of failure. This finding is not indicative of particularly poor outcomes in our institutions-similarly high, and higher rates of up to $92 \%$, have been reported in previous studies. ${ }^{27,28,33,34}$ Since routine replacement is ineffective, research attention should now focus on other interventions to reduce these complications. Improved survival of intravenous catheters for even small increments of time would further reduce the number of insertions, staff workloads, and costs. Improved insertion, securement, and flushing stategies could be key.

Our findings are consistent with previous smaller randomised controlled trials, ${ }^{9-12}$ and a systematic review showed no benefit of routine replacement for phlebitis or catheter-related bloodstream infections (panel). ${ }^{35}$ Thus, much evidence now suggests that clinically indicated replacement is safe. Updated intravenous catheter policies should advocate clinically indicated removal-ie, to monitor and immediately remove intravenous catheters for complications or as soon as treatment is complete. The CDC guidelines already recommend clinically indicated replacement in children, ${ }^{4}$ citing two non-randomised studies (total $\mathrm{n}=589) .{ }^{14}$ Thus, despite a scarcity of large randomised controlled trials, paediatric patients are not subjected to routine replacement, perhaps because of the sensitivities of cannulating children. Our data support extension of these recommendations, and this sensitivity, to the management of adult patients. Insertion of intravenous catheter is painful, requiring the piercing of skin, tissue, and vein with a steel needle at least once, or several times for a difficult insertion. Investigation of patients' perspectives of strategies for replacement of intravenous catheters has been recommended, ${ }^{25}$ but patients are presumably unlikely to want routine replacement since it has no proven benefit. 


\section{Contributors}

CR and JW did the scientific literature search, study design, and grant writing. CR recruited sites, wrote the first and final drafts of the report, and consulted with all authors about the article. MM, who is a statistician, analysed data and prepared figures. All authors contributed to data collection and substantially to interpretation and analysis of data and approved the final version of the report.

\section{Conflicts of interest}

We declare that we have no conflicts of interest.

\section{Acknowledgements}

The Australian National Health and Medical Research Council funded this study through the national competitive grants scheme. We received in-kind support from Griffith University, Royal Brisbane and Women's Hospital, Princess Alexandra Hospital, Gold Coast Hospital, and Monash University. We acknowledge the participants, and the clinical and research staff of the three hospitals. In particular, the research nurses: Kaye Sayed, Catherine Gale, and Wendy Jackman (Royal Brisbane and Women's Hospital); Rebecca Kim, Jan Hine, Joanne Peters, and Mark Wood, (Princess Alexandra Hospital); Kenneth Boag, Tanya Clark, Tracey Patrick, Lyn Jenyns, Julie Pitman, and Melissa Medina (Gold Coast Hospital) for recruitment of patients and data collection. We also thank Nicolas Rossow for development and assistance with the database and David McMillan for microbiology advice. We thank Lukman Thalib and Damhnat McCann for advice on the grant proposal. 


\section{References}

1. Maki DG. Improving the safety of peripheral intravenous catheters. BMJ 2008; 337: 122-23.

2. Zingg W, Pittet D. Peripheral venous catheters: an under-evaluated problem. Int J Antimicrob Agents 2009; 34: S38-42.

3. Maki DG, Kluger DM, Crnich CJ. The risk of bloodstream infection in adults with different intravascular devices: a systematic review of 200 published prospective studies. Mayo Clin Proc 2006; 81: 1159-71.

4. O'Grady NP, Alexander M, Burns LA, et al. Guidelines for the prevention of intravascular catheter-related infections. Clin Infect Dis 2011; 52: e162-93.

5. Cook D, Randolph AG, Kernerman P, et al. Central venous catheter replacement strategies: a systematic review of the literature. Crit Care Med 1997; 25: 1417-24.

6. Bregenzer T, Conen D, Sakmann P, Widmer AF. Is routine replacement of peripheral intravenous catheters necessary? Arch Intern Med 1998; 158: 151-56.

7. Cornely OA, Bethe U, Pauls R, Waldschmidt D. Peripheral Teflon catheters: factors determining incidence of phlebitis and duration of cannulation. Infect Control Hosp Epidemiol 2002; 23: $249-53$.

8. Grune F, Schrappe M, Basten J, Wenchel H, Tual E, Stutzer H. Phlebitis rate and time kinetics of short peripheral IV catheters. Infection 2004; 32: 30-32.

9. Rickard CM, McCann D, Munnings J, McGrail MR. Routine resite of peripheral intravenous devices every 3 days did not reduce complications compared with clinically indicated resite: a randomised controlled trial. BMC Med 2010; 8: 53.

10. Van Donk P, Rickard CM, McGrail MR, Doolan G. Routine replacement versus clinical monitoring of peripheral intravenous catheters in a regional hospital in the home program: a randomized controlled trial. Infect Control Hosp Epidemiol 2009; 30: 915-17. 
11. Webster J, Clarke S, Paterson D, et al. Routine care of peripheral intravenous catheters versus clinically indicated replacement: randomised controlled trial. BMJ 2008; 337: a339.

12. Webster J, Lloyd S, Hopkins T, Osborne S, Yaxley M. Developing a Research base for Intravenous Peripheral cannula re-sites (DRIP trial). A randomised controlled trial of hospital inpatients. Int J Nurs Stud 2007; 44: 664-71.

13. Maki DG, Ringer M. Risk factors for infusion-related phlebitis with small peripheral venous catheters. Ann Intern Med 1991; 114: 845-54.

14. O'Grady NP, Alexander M, Dellinger EP, et al. Guidelines for the prevention of intravascular catheter-related infections. Infect Control Hosp Epidemiol 2002; 23: 759-69.

15. Maki DG, Weise CE, Sarafin HW. A semiquantitative culture method for identifying intravenous-catheter-related infection. N Engl J Med 1977; 296: 1305-09.

16. Thompson SG, Barber JA. How should cost data in pragmatic randomised trials be analysed? BMJ 2000; 320: 1197-200.

17. Maki DG, Ringer M. Evaluation of dressing regimens for prevention of infection with peripheral intravenous catheters: gauze, a transparent polyurethane dressing, and an iodophortransparent dressing. JAMA 1987; 258: 2396-403.

18. Lee WL, Chen HL, Tsai TY, et al. Risk factors for peripheral intravenous catheter infection in hospitalized patients: a prospective study of 3165 patients. Am J Infect Control 2009; 37: 683-86.

19. Mermel LA, Allon M, Bouza E, et al. Clinical practice guidelines for the diagnosis and management of intravascular catheter-related infection: 2009 update by the Infectious Diseases Society of America. Clin Infect Dis 2009; 49: 1-45.

20. Elliott TSJ, Moss HA, Tebbs SE, et al. Novel approach to investigate a source of microbial contamination of central venous catheters. Eur J Clin Microbiol Infect Dis 1997; 16: 210-13.

21. Zhang L, Gowardman J, Rickard C. Impact of microbial attachment on intravascular catheterrelated infections. Int J Antimicrob Agents 2011; 38: 9-15. 
22. Schultz AA, Gallant P. Evidence-based quality improvement project for determining appropriate discontinuation of peripheral IV cannulas. Evid Based Nurs 2005; 8: 8.

23. Palese A, Cassone A, Kulla A, al e. Factors influencing nurses' decision-making process on leaving in the peripheral intravascular catheter after 96 hours: a longitudinal study. J Infus Nurs 2011; 34: 319-26.

24. Bravery K, Dougherty L, Gabriel J, Kayley J, Malster M, Scales K. Audit of peripheral venous cannulae by members of an IV therapy forum. Br J Nurs 2006; 15: 1244-49.

25. Johansson M, Pilhammar E, Willman A. Nurses' clinical reasoning concerning management of peripheral venous cannulae. J Clin Nurs 2009; 18: 366-75.

26. Gaukroger PB, Roberts JG, Manners TA. Infusion thrombophlebitis: A prospective comparison of 645 Vialon and Teflon cannulae in anaesthetic and postoperative use. Anaesth Intensive Care 1988; 16: 265-71.

27. Bausone-Gazda D, Lefaiver CA, Walters C-A. A randomized controlled trial to compare the complications of 2 peripheral intravenous catheter-stabilization systems. J Infus Nurs 2010; 33: 37184.

28. Schears GJ. Summary of product trials for 10,164 patients: comparing an intravenous stabilizing device to tape. J Infus Nurs 2006; 29: 225-31.

29. Gallant P, Schultz AA. Evaluation of a visual infusion phlebitis scale for determining appropriate discontinuation of peripheral intravenous catheters. J Infus Nurs 2006; 29: 338-45.

30. Malach T, Jerassy Z, Rudensky B, et al. Prospective surveillance of phlebitis associated with peripheral intravenous catheters. Am J Infect Control 2006; 34: 308-12.

31. Powell J, Tarnow KG, Perucca R. The relationship between peripheral intravenous catheter indwell time and the incidence of phlebitis. J Infus Nurs 2008; 31: 39-45. 
32. Soifer NE, Borzak S, Edlin BR, Weinstein RA. Prevention of peripheral venous catheter complications with an intravenous therapy team: a randomized controlled trial. Arch Intern Med 1998; 158: 473-77.

33. Chico-Padrón RM, Carrión-García L, Delle-Vedove-Rosales L, et al. Comparative safety and costs of transparent versus gauze wound dressings in intravenous catheterization. J Nurs Care Qual 2011; 26: 371-6.

34. Smith B. Peripheral intravenous catheter dwell times. A comparison of 3 securement methods for implementation of a 96-hour scheduled change protocol. J Infus Nurs 2006; 29: 14-17.

35. Webster J, Osborne S, Rickard C, Hall J. Clinically-indicated replacement versus routine replacement of peripheral venous catheters. Cochrane Database Syst Rev 2010; 17: CD007798. 